\title{
METAL BINDING IN AMYLOID BETA-PEPTIDES SHOWS \\ INTRA- AND INTER-PEPTIDE COORDINATION MODES
}

Francesco Stellato , Gianfranco Menestrina ${ }^{2 \$}$, Mauro Dalla Serra², Cristina Potrich², Rossella Tomazzolli $^{2}$, Wolfram Meyer-Klaucke ${ }^{3}$, and Silvia Morante ${ }^{1 *}$

${ }^{1}$ Dipartimento di Fisica, Università di Roma “Tor Vergata" INFM and INFN, Via della Ricerca Scientifica 1, I-00133 Roma, Italy

${ }^{2}$ Consiglio Nazionale delle Ricerche - Istituto Trentino di Cultura, Istituto di Biofisica - Sezione di Trento, Via Sommarive 18, I-38050 Povo, Italy

${ }^{3}$ European Molecular Biology Laboratory, c/o DESY, Notkestrasse 85, D-22603 Hamburg, Germany

Running Title: Metal Binding in Amyloids

${ }^{\$}$ The work presented in this paper started with the invaluable collaboration of G. Menestrina and we would like to dedicate it to his memory.

*Address correspondence to: Silvia Morante, Dipartimento di Fisica, Università di Roma "Tor Vergata", Via della Ricerca Scientifica, 00133 Roma, Italy, Tel. +39-0672594554; Fax +39062025259; E-Mail: morante@roma2.infn.it. 


\begin{abstract}
X-ray absorption spectroscopy data show different metal binding site structures in $\beta$-amyloid peptides according to whether they are complexed with $\mathrm{Cu}^{2+}$ or $\mathrm{Zn}^{2+}$ ions. While the geometry around copper is stably consistent with an intra-peptide binding with three metal-coordinated Histidine residues, the zinc coordination mode depends on specific solution conditions. In particular, different sample preparations are seen to lead to different geometries around the absorber that are compatible with either an intra- or an inter-peptide coordination mode. This result reinforces the hypothesis that assigns opposite physiological roles to the two metals, with zinc favoring peptide aggregation and, as a consequence, plaque formation.
\end{abstract}




\section{INTRODUCTION}

Amyloidosis is a family of pathologies caused by the transition of endogenous proteins and peptides from the physiological globular configuration to a pathological fibrillar state. The term describes a heterogeneous group of diseases (more than 20), which are characterized by extra-cellular deposition of fibrillar material (Pepys 2001). Among them the Alzheimer's disease $\left(\mathrm{AD}^{1}\right)$ is a progressive and devastating neuro-degenerative pathology affecting an important fraction of the aged population in the developed world (Selkoe 2001). AD is characterized by memory disorders, degradation of the personality and other behavioral abnormalities correlated to the loss of neurons from cortex and hippocampus. These events are accompanied by peculiar morphological manifestations like, formation of senile plaques in the brain, amyloidosis of brain vessels and intra-neuronal deposits of amyloid fibrils. The major component of the $\mathrm{AD}$ amyloid plaques are the $\beta$-amyloid peptides (A $\beta$ ). $\mathrm{A} \beta$-peptides are produced by two enzymes ( $\beta$ - and $\gamma$-secretase) that act by proteolytic cleavage of a transmembrane protein, called $\beta$-amyloid precursor protein (A $\beta P P)$. The function of $A \beta P P$, which is expressed in virtually all animal cells, is currently unknown. Cleavage of A $\beta P P$ takes place at the Cterminal end, at heterogeneous positions, producing peptides of variable length from 39 to 43 residues. The two most frequently produced peptides have either $40\left(\mathrm{~A} \beta_{1-40}\right)$ or $42\left(\mathrm{~A} \beta_{1-42}\right)$ residues. In the extra-cellular space soluble $A \beta$-peptides assembles into small neuro-toxic aggregated fibrils. Fibrillar $A \beta_{1-42}$ is preferentially deposited in neuritic plaques, whereas fibrillar $A \beta_{1-40}$ is more commonly present on vascular walls (Selkoe 2001).

It has been observed that plaques contain large amounts of transition metals like $\mathrm{Cu}, \mathrm{Fe}$ and $\mathrm{Zn}$ (the last one being the most abundant). Their role is not yet fully understood, but it has been conjectured to be crucial in the pathological effects of AD (Huang et al. 1977; Bush 2000; Miura et al. 2000; Finefrock et al. 2003; Karr et al. 2004). The interest of elucidating the role of metals in the development of the disease strongly increased after noticing that $\mathrm{Cu}^{2+}$ and $\mathrm{Zn}^{2+}$ chelators can be used to solubilize $\mathrm{A} \beta$ 
aggregates (Cherny et al. 2001; Opazo et al. 2003). Both $\mathrm{Cu}^{2+}$ and $\mathrm{Zn}^{2+}$ ions are known to promote aggregation (Tjernberg et al. 1999) but, besides $\mathrm{Zn}^{2+}$ being always more effective than $\mathrm{Cu}^{2+}$ (Comai et al. 2003), an inhibitory effect of $\mathrm{Cu}^{2+}$ on the aggregation inducing effect of $\mathrm{Zn}^{2+}$ has been observed (Suzuki et al. 2001). Two distinct ways in which the metal ion can bind the peptide have been suggested (Miura et al. 2000; Curtain et al. 2001) which are generically termed inter- and intramolecular modes, respectively. In the inter-molecular mode $A \beta$-peptides are supposed to be crosslinked with "A $\beta$-metal-A $\beta$ " bridges, while in the intra-molecular mode the atoms participating to the metal coordination all belong to the same peptide. Different structural models for each of the two binding modes have been proposed in which different numbers of Histidine residues are involved in the metal coordination.

X-ray Absorption Spectroscopy (XAS) can be profitably used for structural studies on biological material, as the technique can be employed for samples in any state of aggregation, in particular for proteins in solution, thus allowing investigations in physiological conditions. Owing to its chemical selectivity and sensitivity to the local atomic arrangement around the absorber, one can get a clear-cut identification of the amino acid residues primarily bound to the metal (Lee et al. 1981; Meneghini and Morante 1998; Morante et al. 2004; Redecke et al. 2005).

We have used XAS to investigate the local structure around the ion in samples of A $\beta$-peptides complexed with $\mathrm{Cu}^{2+}$ or $\mathrm{Zn}^{2+}$. We will show in this paper that an accurate analysis of their XAS spectra reveals that copper and zinc acquire distinct types of coordination geometries. These geometries can be characterized by the number of the Histidine imidazole rings directly coordinated to the metal. Our findings point to the possibility that $\mathrm{Cu}^{2+}$ and $\mathrm{Zn}^{2+}$ may play a different, possibly antagonistic, role in amyloid aggregation.

MATERIALS AND METHODS 
Sample preparation - The $\mathrm{Cu}^{2+}$ - and $\mathrm{Zn}^{2+}-\mathrm{A} \beta_{1-40}$ complexes subjected to XAS measurements in this investigation have been prepared according to the following protocols. Synthetic $A \beta_{1-40}$ peptide has been purchased from BioSource International (CA, USA). They have a purity index $>96 \%$. The primary amino acidic sequence of the peptide is

$\mathrm{H}_{3} \mathrm{~N}^{+}$-DAEFRHDSGYEVHHQKLVFFAEDVGSNKGAIIGLMVGGVV-COO-

Aliquots of $A \beta_{1-40}$ peptides, solubilized in hexafluoroisopropanol (Sigma), were dried under nitrogen flux and re-suspended in a $20 \mathrm{mM}$ Tris $/ \mathrm{HCl}$ buffer, $\mathrm{pH}=7$, to obtain a final $0.4 \mathrm{mM}$ peptide concentration. $\mathrm{Cu}^{2+}$ or $\mathrm{Zn}^{2+}$ complexes are prepared, starting from $\mathrm{CuSO}_{4}$ and $\mathrm{ZnCl}_{2}$ respectively, in three different metal/peptide concentration ratios, $0.5,1$ and 2 .

The over-stoichiometric solution (i.e. the one with metal/peptide concentration equal to 2) has been used only to prepare the re-suspended pellet solution. The reason for this is that in the other preparations we wanted to avoid that some amount of the metal could remain free in solution, something which is unsuited for XAS measurements. Within the procedure used to prepare the resuspended pellet a possible excess of metal in solution is removed together with the supernatant after the centrifugation step (see below).

A fraction of the prepared solutions was directly transferred in a 1-mm thick plastic holder closed by two Kapton windows, rapidly frozen in liquid nitrogen and immediately brought to measurement. The rest was incubated overnight at $37^{\circ} \mathrm{C}$. After the incubation, samples are centrifuged at $4^{\circ} \mathrm{C}$ for $1 \mathrm{~h}$ at $133000 \mathrm{~g}$ (Airfuge Ultracentrifuge Beckman, equipped with the $30^{\circ}$ angle A-100 rotor). This procedure was devised to favor a possible aggregation among peptides. The resulting pellets are re-suspended in the buffer, and transferred into the sample holder, rapidly frozen and measured. In order to check whether any amount of dissolved peptide is removed together with the supernatant, the absorbance spectrum of the supernatant at $220 \mathrm{~nm}$ is measured. No detectable signal is found. 
All five samples (two solutions at metal/peptide concentration ratios 0.5 and 1 and three re-suspended pellets) for each type of metal have been subjected to XAS measurements.

$X A S$ data collection - XAS data at the $\mathrm{Cu}^{2+}$ and $\mathrm{Zn}^{2+} \mathrm{K}$-edge have been collected at the $\mathrm{D} 2$ bending magnet beam line of the EMBL Outstation Hamburg at DESY. The synchrotron was operating at 4.5 $\mathrm{GeV}$ with ring currents ranging from 90 to $149 \mathrm{~mA}$. A Si[111] double crystal mono-chromator and a focusing mirror with a cut-off energy of $21.5 \mathrm{KeV}$ were used throughout the study. The X-ray spectra of the samples were recorded in fluorescence mode using a 13-element Ge solid-state detector (Canberra, Meriden, CT).

Data analysis - Data collected ${ }^{2}$ in each run are first averaged and, after background subtraction, the resulting spectra are normalized using the EXPROG package (Nolting and Hermes 1992). EXAFS (Extended X-ray Absorption Fine Structure) data are analyzed and fitted using the EXCURV98 package (Binsted et al. 1992; Binsted 1998).

In the following we shall discuss the data of only three samples. In fact, the XAS spectra of all the five different kinds of $\mathrm{Cu}^{2+}$ samples (i.e. direct solution and re-suspended pellet in different metal/peptide ratios) show absorption spectra that are indistinguishable within experimental data fluctuations. As for $\mathrm{Zn}^{2+}$ complexes, a sensible difference in the spectral features between solution and pellet samples is visible. No difference is seen instead when spectra of samples obtained from different initial metal/peptide concentration ratio are compared. Consequently, we have subjected to a detailed analysis only the XAS spectra of the following samples:

1) $\mathrm{Cu}^{2+}$ complex in solution with metal/peptide ratio equal to $1-(\mathrm{Cu}-\mathrm{A} \beta)_{1}$ from now on.

2) $\mathrm{Zn}^{2+}$ complex in solution with metal/peptide ratio equal to 1 - $(\mathrm{Zn}-\mathrm{A} \beta)_{1}$ from now on. 
3) $\mathrm{Zn}^{2+}$ complex, re-suspended pellet, with (initial) metal/peptide ratio equal to $1-(\mathrm{Zn}-\mathrm{A} \beta)_{2}$ from now on.

We also discuss the spectra of rapidly frozen $0.4 \mathrm{mM} \mathrm{Cu}$ and $\mathrm{Zn}$ buffer solutions in the absence of peptide (in the following, Cu-buffer and Zn-buffer, respectively).

Model building - It is well known that to get reliable information from XAS data, a crucial point of the analysis is the choice of the initial trial geometry around the absorber. Lacking crystallographic data for A $\beta$-peptides, the only indication about possible metal ligands is the one that comes from the knowledge of the peptide amino acidic sequence. Thus in order to start the fitting procedure from realistic geometries, we followed a procedure suggested by (Cheung et al. 2000). We have submitted the normalized spectra, as they have emerged after the previously described data processing, to a first coarse-grain refinement using as initial atomic coordinates different sets of crystallographic data chosen among those available in the Metallo-protein Database and Browser (MDB) data collection (Castagnetto et al. 2002). Of the many structures we tried we have selected as starting geometry the one around the active site of the $\mathrm{Cu}^{2+}$ derivative of the Astacin Zn-endopeptidase (PDB I.D.: 1IAA). In this protein $\mathrm{Cu}^{2+}$ is penta-coordinated to three Histidine residues, one Tyrosine residue and one water molecule. The PDB files that were finally used as inputs to be read by Excurv98 for the successive fit were further manually adjusted case by case to match as accurately as possible the peculiar overall structure of the metallic site of each one of our individual samples. In particular, for the sample $(\mathrm{Cu}-$ $\mathrm{A} \beta)_{1}$ the starting geometry has been modified by adjusting the Histidine and Tyrosine relative plane orientation. In the cases of samples $(\mathrm{Zn}-\mathrm{A} \beta)_{1}$ and $(\mathrm{Zn}-\mathrm{A} \beta)_{2}$, after the obvious substitution of the absorbing atom from $\mathrm{Cu}^{2+}$ to $\mathrm{Zn}^{2+}$, further adjustments have been made which consisted in changing both the metal coordination number as well as the number of bound Histidine residues to best cope with the geometrical structure of the atomic Zn environment seen in the data. 
Following (Binsted et al. 1992), we have chosen to treat as a rigid body the internal geometry of the Histidine imidazole ring as well as that of the Tyrosine phenyl ring. As a result, the remaining free degrees of freedom of the Histidine and Tyrosine metal bound residues are the distance between the bound nitrogen of the imidazole ring and the metal (Metal- $\mathrm{N}_{\text {imidazole }}$ bond) and the distance between the bound oxygen of the Tyrosine hydroxyl group and the metal (Metal- $\mathrm{O}_{\text {hydroxyl }}$ bond), respectively. To these one has to add the bond angle centered on the oxygen of Tyrosine hydroxyl. In the future we will often refer to the light atoms through which the residue is bound to the absorber as the "leading" residue atoms. Furthermore in the fit, both imidazole and phenyl rings are let free to (rigidly) rotate in all directions. Indeed, in the final step of the fitting procedure, when also Multiple Scattering (MS) contributions coming from inter-ligand photo-electron paths are considered, the relative imidazoleimidazole and imidazole-phenyl ring orientations become relevant.

The reason for including MS contributions, which are usually small in the EXAFS region, is that this is not so for the MS contributions coming from (almost) collinear structures, as they are strongly enhanced by the large forward scattering amplitude resulting from their peculiar geometric arrangement (focusing effect) $)^{3}$.

It is important to observe that in general one cannot distinguish among light scatterers (like $\mathrm{N}$ and $\mathrm{O}$ ) only on the basis of their individual contribution to the EXAFS signal. In the situation like the present one, where they play the role of leading residue atoms, they can be however unambiguously identified because they act as a tag for the presence of imidazole or phenyl rings.

As for the statistical significance of our fits, we have checked that in all cases the number of independent data points ${ }^{4}, N_{\text {ind }}$, is comfortably larger that the number of fitted parameters, $N_{\text {par }}$. 


\section{RESULTS AND DISCUSSION}

In discussing the features of XAS spectra it is useful to analyze the low energy X-ray absorption near edge (XANES) and the high energy EXAFS regions separately.

The XANES region - The structure of the XANES region is in principle fairly sensitive to the electronic structure of the absorber and the symmetry of the local environment around it. However, due to the non-negligible effect of very complicated electronic processes (like transitions to bound states, multiple scattering events, etc.), a quantitative interpretation of this part of the spectrum is very problematic (Lee and Pendry 1975; Benfatto et al. 1986; Gurman et al. 1986; Koningsberger and Prins 1988; Rehr and Albers 1990). Despite this difficulty, the comparison of XANES spectra of structurally similar samples can yield valuable information on similarities and differences between relative local geometries (Bianconi et al. 1986).

Fig.1

In Fig. 1 we compare the absorption coefficient, $\mu$, in the XANES spectral region of the A $\beta_{1-40}$ peptide complexed with either $\mathrm{Cu}^{2+}$ or $\mathrm{Zn}^{2+}$. In the figure data for $(\mathrm{Cu}-\mathrm{A} \beta)_{1}$ (dotted line), $(\mathrm{Zn}-\mathrm{A} \beta)_{1}$ (broken line), and $(\mathrm{Zn}-\mathrm{A} \beta)_{2}$ (continuous line) samples are plotted together.

The three spectra show significant differences, suggestive of different arrangements of near scatterers around the metallic absorber. The most visible differences are in the rising of the slope around the edge and in the shape of the first peak soon after the edge (the so-called white line).

With this in mind, we have compared in Fig. 2 the edge region of the three $\mathrm{Zn}$ samples, $(\mathrm{Zn}-\mathrm{A} \beta)_{1},(\mathrm{Zn}-$ $\mathrm{A} \beta)_{2}$ and $\mathrm{Zn}$-buffer (dotted, broken and continuous line respectively). Concentrating attention on the shape of the white line, we see that the peak symmetry progressively lowers moving from the $\mathrm{Zn}$ buffer, to the $(\mathrm{Zn}-\mathrm{A} \beta)_{1}$ sample and from the latter to the $(\mathrm{Zn}-\mathrm{A} \beta)_{2}$ sample, thus suggesting (Natoli and Benfatto1986; Tyson et al. 1992) a progressive loss of symmetry of the geometry around the absorber. 
The EXAFS region - In the so-called EXAFS region, which extends from about $50 \mathrm{eV}$ above the edge energy onward, valuable quantitative structural information can be extracted from experimental data, even starting with limited information on the atomic structural environment around the metal. We first discuss the $\mathrm{A} \beta$-amyloid samples complexed with $\mathrm{Cu}$ and then those complexed with $\mathrm{Zn}$.

Fig.3

Cu-samples - Fig.3 shows the results of the fit to the EXAFS spectrum of the $(\mathrm{Cu}-\mathrm{A} \beta)_{1}$ sample. The simulated (i.e. theoretical) signal (black line) is seen to reproduce quite satisfactorily the experimental signal (gray line). The experimental data for the structural signal ${ }^{2}, \chi(k)$, as well as the simulated signal, have been weighted by a factor $k^{3}$ (Teo and Lee 1979) to compensate for their rapid decrease with increasing $k$.

In the structural model which best fits the data we find that $\mathrm{Cu}^{2+}$ is bound to a Tyrosine residue (possibly Tyr10), three Histidine residues (supposedly all belonging (Curtain et al. 2001) to the same peptide, namely His6, His13 and His14) and an $\mathrm{OH}^{-}$(water) in a penta-coordinated structure. We observe that a good fit to the detailed features of the data could only be obtained by allowing the simulated signal to include inter-ligand MS contributions. Their magnitude significantly depends on the relative orientation of the planes in which imidazole and phenyl rings lie and on the bond angle centered on the oxygen of the Tyrosine.

It should be observed that in some recent papers (see Kowalik-Jankowska et al. 2003, Syme et al. 2004, Karr et al. 2005) EPR measurements performed on $A \beta$-peptides of different lengths, including $A \beta_{1-40}$, show no evidence for the Tyr10 coordination to $\mathrm{Cu}$, at variance with what we find from the best fit to our data. Discrepancies of this kind are not of fundamental relevance for our analysis. For us the key point is the number of coordinated Histidines (which is always well established, Meneghini \& Morante, 1998) and the fact that all the Cu-samples have identical (within errors) EXAFS spectra, independently of their initial peptide/metal concentration and preparation procedure. 
$Z n$-samples - At variance with the case of the $\mathrm{Cu}^{2+}-\mathrm{A} \beta_{1-40}$ complexes, the spectra of the $\mathrm{Zn}^{2+}-\mathrm{A} \beta_{1-40}$ complexes, obtained by the two different preparation procedures described above (i.e., either by direct dissolving the peptide in the buffer - sample $(\mathrm{Zn}-\mathrm{A} \beta)_{1}$ - or by re-suspending the pellet - sample (Zn$\mathrm{A} \beta)_{2}$ - (see MATERIALS AND METHODS), appear substantially different from each other. Fig.4 Looking first at the spectrum of the sample (Zn-A $)_{1}$ (Fig.4A), it is pretty evident that the amplitude of the EXAFS oscillations decrease with increasing $k$ more slowly than one expects when only light scatterers are assumed to surround the absorber. This fact can be taken as an indication for the presence of "heavier" (than $\mathrm{C}, \mathrm{N}$, and $\mathrm{O}$ ) nearby scatterers.

To try to reproduce this feature of the data, different structural models involving heavy atoms, have been considered and tested. Among others, we have examined the possibility that the involved heavy atom might be a sulfur (S), belonging to the lateral chain of the methionine residue (Met35) ${ }^{5}$. This hypothesis has been, however, ruled out by a detailed analysis of the data. The presence of $\mathrm{S}$ is compatible with our XAS results neither if located at a first nor at a second shell distance. In fact, in both cases we find that the Fourier transforms (FT) of the fitted curves (data not shown) do not satisfactorily reproduce the structural features seen in the data.

The fit shown in Fig.4A is obtained instead by allowing two chlorine ions $\left(\mathrm{Cl}^{-}\right)$to belong to the first coordination shell. We recall that $\mathrm{Cl}^{-}$ions were present in the buffer in which the peptides have been dissolved. Comparing with the situation where a $\mathrm{S}$ is present we get a fit with a definitely better $R$ value $^{7}$ and smaller errors on the fitted parameters, as well as a rather good representation of the FT of the data (see below Fig.6, panel b).

In Fig.4B we show the experimental (gray line) and simulated (black line) XAS spectrum of the sample $(\mathrm{Zn}-\mathrm{A} \beta)_{2}$. A difference between the shape of this spectrum and that of the $(\mathrm{Zn}-\mathrm{A} \beta)_{1}$ sample is seen in the pronounced broadening of the first peak. 
Description of the Table - In the Table we report the values of the best fit parameters, defining the geometry of the atomic structure around the metal, for each of the samples we have analyzed. They $\operatorname{are}^{6}$ : type and number [first and second column] of the coordinated chemical group (atom or residue); distance from the absorber [third column] of the coordinated atom or of the leading atom of the coordinated residue; value of the corresponding DW width, $\sigma_{D W}^{2}$ [fourth column]. Errors are purely statistical and are computed from the knowledge of the correlation matrix provided by EXCURV98. In the last row we report the Fermi energy shift $\Delta E_{f}$ and the $R$-factor of the fit ${ }^{7}$, as well as the number of independent data points, $N_{\text {ind }}$ (see note ${ }^{4}$ ), and the number of fitted parameters, $N_{p a r}$.

Table

We stress again that, even differences in the coordination distance of the metal bound leading atoms which may seem "not too large", on the basis of standard considerations, are significant here, as any modification of this distance, by dragging along with it all the atoms of the residue ring, significantly alters the magnitude of this bulk-contribution (mostly coming from MS events) to the signal. Thus even minor modifications of some of the parameters characterizing the overall amino acid positions in space largely affects the quality of the fit.

$\mathrm{Cu}$-site geometry - As we previously mentioned, it turns out from our fit to the data that $\mathrm{Cu}$ in $(\mathrm{Cu}-\mathrm{A} \beta)_{1}$ is penta-coordinated to three nitrogens, belonging to three Histidine residues, and two oxygens, one belonging to the oxygen of a Tyrosine residue (the latter could be Tyr10) and the remaining one possibly belonging to either $\mathrm{OH}^{-}$(water) or to some amino acidic residue different from the bound Histidine and Tyrosine.

Notice that we have grouped together two coordinated Histidines because they are found to be indistinguishable within errors from one another. They in fact lie at the same distance from the absorber and have the imidazole rings similarly oriented. 
Zn-site geometry - Unlike $\mathrm{Cu}, \mathrm{Zn}$ in both $(\mathrm{Zn}-\mathrm{A} \beta)_{1}$ and $(\mathrm{Zn}-\mathrm{A} \beta)_{2}$ samples is hexa-coordinated. The Table shows that in $(\mathrm{Zn}-\mathrm{A} \beta)_{1}$ there are two $\mathrm{Cl}^{-}$ions located at $2.3 \AA$ from the absorber. In the first $\mathrm{Zn}$ coordination shell one also finds one $\mathrm{N}$ atom belonging to a Histidine residue and three oxygen, one belonging to the oxygen of a Tyrosine residue and the remaining two possibly belonging, as in the case of the $(\mathrm{Cu}-\mathrm{A} \beta)_{1}$ sample, either to water or to amino acidic residues other than Histidine and Tyrosine. An observation is worth here. Surely enough the presence of a high concentration of $\mathrm{Cl}^{-}$ions is not a physiological situation. Our finding is nevertheless useful as it allows us to argue that their presence in the neighboring of the metal is an indirect evidence for the propensity of the system towards quite an "open" geometry. An open geometry is also suggested by the fact that only a single Histidine residue is bound to $\mathrm{Zn}$, at variance with the situation we discovered in the case of the $\mathrm{Cu}$ complexes, where three Histidine residues were present in the metal coordination shell.

This kind of model is confirmed by the observation that the values of the fit parameters obtained for the $(\mathrm{Zn}-\mathrm{A} \beta)_{2}$ sample are suggestive of an intermediate situation between the "open" geometry of $(\mathrm{Zn}-\mathrm{A} \beta)_{1}$ and the more "compact" one of $(\mathrm{Cu}-\mathrm{A} \beta)_{1}$. In the $(\mathrm{Zn}-\mathrm{A} \beta)_{2}$ case, in fact, as shown in Table, the $\mathrm{Zn}$ hexa-coordination mode is obtained by two $\mathrm{N}$ atom belonging to two Histidine residues and four oxygen, one belonging to the oxygen of a Tyrosine residue and the remaining three either to water or to amino acidic residues other than Histidine and Tyrosine residues, as before. The intermediate $(\mathrm{Zn}-\mathrm{A} \beta)_{2}$ geometry is suggested by the presence of two Histidine residues, instead of only one as in the (Zn-A $\beta)_{1}$ case, and by the fact that no $\mathrm{Cl}^{-}$are present in the first coordination shell, indicating that the solvent could not access the absorber environment.

Buffers - For completeness, also the parameters of the best fit of the two $\mathrm{Cu}^{2+}$ and $\mathrm{Zn}^{2+}$ buffer samples are reported in the Table. In the absence of peptide $\mathrm{Cu}^{2+}$ is tetra-coordinated to four oxygen atoms (Op 
in the Table) with a planar structure. Two further oxygen atoms are found at a larger distance possibly in an axial position (Oa in the Table). In the Zn-buffer the best fit parameters are compatible with a (slightly distorted) octahedral geometry (D’Angelo et al. 2002).

Structural models - The molecular arrangement around the absorber, as it comes out from the file in PDB (Protein Data Bank) format directly generated by EXCURV98, is sketched in Fig.5 in the three cases we have studied, namely $(\mathrm{Cu}-\mathrm{A} \beta)_{1},(\mathrm{Zn}-\mathrm{A} \beta)_{1}$ and $(\mathrm{Zn}-\mathrm{A} \beta)_{2}$ in panels $\mathrm{A}, \mathrm{B}$ and $\mathrm{C}$, respectively. The structures are shown mainly to give an immediate idea of the relative position and orientation of imidazole and phenyl rings.

Fig.5

Panel A shows a cartoon of the molecular environment around $\mathrm{Cu}$. It is important to appreciate the compact shape of the structure around the metal. In fact, two of the three imidazole rings (those indicated by His1 and His2 in the Table) lie almost in the same plane with their normals forming an angle ${ }^{8}$ of about $20^{\circ}$, while the normal to the imidazole plane of His 3 lies almost perpendicular to the common plane of the imidazole rings of His 1 and His2. The other interesting parameter is the bond angle $\mathrm{Cu}-\mathrm{O}(\mathrm{Tyr})-\mathrm{C}(\mathrm{Tyr})$. Its best fit value is $113^{\circ}$.

In the case of the $(\mathrm{Zn}-\mathrm{A} \beta)_{1}$ complex (panel $\left.\mathrm{B}\right)$, the angle between the normal to the phenyl Tyrosine plane and the normal to the imidazole Histidine plane is $65^{\circ}$, while the $\mathrm{Zn}-\mathrm{O}(\mathrm{Tyr})-\mathrm{C}(\mathrm{Tyr})$ bond angle turns out to be $120^{\circ}$.

The molecular arrangement around $\mathrm{Zn}^{2+}$ in the $(\mathrm{Zn}-\mathrm{A} \beta)_{2}$ complex (panel C), shows that the imidazole rings of the two Histidine residues lie within errors in the same plane, while the phenyl plane of the Tyrosine is perpendicular to the common plane of the two imidazole rings. As for the $\mathrm{Zn}-\mathrm{O}$ (Tyr)$\mathrm{C}(\mathrm{Tyr})$ bond angle, it comes out to be again $120^{\circ}$. 
Comment - A final general comment is worth making about the overall quality of our fits. As discussed in the Introduction, a fit should be regarded as sufficiently good if the value of the $R$-factor, defined in ${ }^{7}$, lies between $20 \%$ and $40 \%$. In all cases we analyzed we have obtained values of $R$ between $25 \%$ and $35 \%$ (see Table) which we interpret as a strong indication of a fairly satisfactory agreement between experimental data and theoretical interpretation. It should be noted that, though superficially the fits to $\mathrm{Cu}^{2+}$ data look nicer than what might seem in the case of the $\mathrm{Zn}^{2+}$ samples, the value of the $R$-factor is somewhat higher in the former case than in the latter. This fact is to be ascribed to the higher noise level of the $\mathrm{Cu}$ data at high $k$. We cannot provide a straightforward explanation for this behavior. It is the result of many different effects, possibly including the better performance of the beam-line at the Zn energy edge.

Fourier Transform - A useful check of the quality of our fits may be obtained by comparing the FT of the experimental EXAFS data with the FT of the fitted theoretical signal. This comparison is shown in Fig.6. We report the FT of the EXAFS spectra of the three samples, $(\mathrm{Cu}-\mathrm{A} \beta)_{1},(\mathrm{Zn}-\mathrm{A} \beta)_{1}$, and $(\mathrm{Zn}-\mathrm{A} \beta)_{2}$, in panels A, B and C, respectively with superimposed the FT of the fitted theoretical signal. It can be appreciated that the characteristic peaks present in the FT of the experimental data (gray line) are satisfactorily reproduced by the behavior of the theoretical curve (black line). This level of consistency is particularly significant, because, we recall, what we have been fitting in our analysis was not the FT of the data, but directly the structural signal $\chi(\boldsymbol{k})$ in $k$ space $^{2}$.

Fig. 6

\section{CONCLUSIONS}

There exist in the literature conflicting statements about the role of $\mathrm{Cu}^{2+}$ and $\mathrm{Zn}^{2+}$ ions in providing protection against or act as promoters of plaques formation and the question of whether the binding of metals might help enhancing or quenching AD development is still awaiting for a firm answer. This 
state of affairs makes the structural characterization of the binding site of $\mathrm{Cu}$ and $\mathrm{Zn}$ a very interesting field of investigation, especially in view of possible development of effective therapeutic strategies. In this paper we have studied the coordination mode of $\mathrm{Cu}-\mathrm{A} \beta$ and $\mathrm{Zn}-\mathrm{A} \beta$ complexes. Already the qualitative analysis of XANES region of the data (Fig.1) suggests significant differences in the geometrical structure around the metal when $\mathrm{Cu}$ and $\mathrm{Zn}$ samples are compared. A rather precise analysis of the atomic environment around the coordination site has been then achieved by the quantitative results we have obtained from a fit to the EXAFS region of the spectrum (Fig.3, Fig.4, and Table). Visible differences between the EXAFS spectra of $\mathrm{Zn}$ and $\mathrm{Cu}$ samples can be interpreted as suggestive for a different role played by the two ions. Our findings are consistent with indications, already existing in the literature (Miura et al. 2000; Curtain et al. 2001), that metals can bind A $\beta$ peptides in an intra- as well as in an inter-peptide coordination mode.

More precisely, we have found that the coordination mode of the metal in the $\mathrm{Cu}^{2+}-\mathrm{A} \beta_{1-40}$ complex, with three Histidine residues and a Tyrosine residue (all possibly belonging to the same peptide, i.e. His6, His13, His14 and Tyr10), is compatible with an intra-peptide coordination mode in agreement with the model suggested in (Suzuki et al. 2001). Furthermore, the fact that the spectra of the $\mathrm{Cu}$ complexes coming from different preparations are essentially identical is strongly suggestive of a rather stable metal-peptide arrangement that does not seem to change when we submit the sample to a preparation procedure (from solution to pellet) that is supposed to favor peptide aggregation.

At variance with the situation we described for the Cu-peptide complexes, the geometrical arrangement around the metal in $\mathrm{Zn}^{2+}-\mathrm{A} \beta_{1-40}$ complexes is significantly modified under a change of the preparation protocol. In particular, the presence of $\mathrm{Cl}^{-}$ions in the first coordination shell, required when fitting the EXAFS region of the spectrum of the $(\mathrm{Zn}-\mathrm{A} \beta)_{1}$ complex, is a clear indication of a metal site easily accessible to the solvent or, in other words, of a quite "open" geometrical structure around the $\mathrm{Zn}^{2+}$ ion. 
This "open" structure is partially lost in the re-suspended pellet preparation of the $\mathrm{Zn}^{2+}-\mathrm{A} \beta_{1-40}$ sample. Consistently, the best fit structure of the latter excludes the presence of coordinated $\mathrm{Cl}^{-}$ions.

The two $\mathrm{Zn}^{2+}-\mathrm{A} \beta_{1-40}$ complexes also differ in the number of Histidine residues bound to the metal. This number is two for the $(\mathrm{Zn}-\mathrm{A} \beta)_{2}$ complex and only one for the $(\mathrm{Zn}-\mathrm{A} \beta)_{1}$ complex. The presence of two bound Histidine residues is compatible with a model in which the metal plays the role of a bridge between the imidazole rings of two Histidine residues possibly belonging to different peptides in a "A $\beta-Z n-A \beta$ " configuration. This configuration is precluded to the $(Z n-A \beta)_{1}$ complex because here only one Histidine residue appears to be coordinated to the metal.

We interpret these results as evidence for the fact that, owing to the peculiarity of the pellet preparation, the Zn-peptide complex undergoes a structural modification which favors an inter-peptide aggregation process. We recall again that this is in contrast with what we find for the metal coordination mode in the Cu-peptide complex whose structure is insensitive to the preparation procedure.

It should be immediately said that only on the basis of the results of the fit one cannot rule out the possibility that the two Histidine residues bound to $\mathrm{Zn}^{2+}$ in the ( $\left.\mathrm{Zn}-\mathrm{A} \beta\right)_{2}$ complex belong to the same peptide. In principle we might be in the presence of a mixed geometry in which some of the $\mathrm{Zn}$ ions bind Histidine residues of different peptides with some others binding Histidine residues belonging to the same peptide. We have, however, a further important piece of information which definitely points in the direction of an inter-peptide aggregation geometry for the pellet sample $(\mathrm{Zn}-\mathrm{A} \beta)_{2}$. This comes from the comparison of the normalized total fluorescence counts associated to the spectral measurements of the two types of $\mathrm{Zn}$-complexes, which were taken under the same experimental conditions. One finds that in the case of the $(\mathrm{Zn}-\mathrm{A} \beta)_{2}$ sample the number of counts is by about a factor of 2 smaller than what one measures in the case of the $(\mathrm{Zn}-\mathrm{A} \beta)_{1}$ sample. This means that the actual $\mathrm{Zn}$ 
concentration in $(\mathrm{Zn}-\mathrm{A} \beta)_{2}$ is about half the concentration in $(\mathrm{Zn}-\mathrm{A} \beta)_{1}$, although both were prepared starting from identical metal concentrations. This fact is fully consistent with the assumed aggregation process, supposedly taking place in the re-suspended pellet preparation of the $(\mathrm{Zn}-\mathrm{A} \beta)_{2}$ sample. In this case, in fact, each $\mathrm{Zn}$ atom is bound to a pair of peptides (the probability of multiple cross linkage being negligible). The left-over unbound $\mathrm{Zn}$ atoms are essentially free and are removed with the supernatant before the pellet is re-dissolved. As it was remarked above in the Sample preparation paragraph, there is no detectable amount of free peptide in the supernatant. This means that the whole amount of dissolved peptide is left out in the pellet.

The overall situation is schematically depicted in Fig.7 where a suggestive illustration of the three possible structures is presented. In panel A the structure of the $(\mathrm{Cu}-\mathrm{A} \beta)_{1}$ complex is shown. Here the metal is tightly bound to three Histidines (only bonds with Histidine residues are explicitly drawn) in a fairly closed structure, which "protects" the metal against any further interaction. Panel B shows the open structure of the $(\mathrm{Zn}-\mathrm{A} \beta)_{1}$ complex (a single Histidine residue is bound) that, making available the metal for further interaction, may switch (under appropriate physico-chemical conditions) to the "A $\beta$ $\mathrm{Zn}-\mathrm{A} \beta$ " configuration of the $(\mathrm{Zn}-\mathrm{A} \beta)_{2}$ complex shown in panel C.

In conclusion we would like to stress that, besides a direct proof of the existence of different coordination modes of Metal-A $\beta$ complexes, our work yields significant insights on the specificity of the Metal- $\mathrm{A} \beta$ interaction and suggests differences in the structural conformation of the complex that depend on the nature of the coordinated metal.

Acknowledgements - We are very grateful to G.C. Rossi for discussions and a careful reading of the manuscript. We would also like to thank G. La Penna for useful suggestions and discussions. 


\section{FOOTNOTES}

This work was partly supported by INFM, INFN, CNR, ITC and the: "European Community-Research Infrastructure Action under the FP6 "Structuring the European Research Area Programme" contract number RII3/CT/2004/5060008.

${ }^{1}$ The abbreviations used are: $\mathrm{AD}$, Alzheimer's disease; $\mathrm{A} \beta$, amyloid $\beta$-peptide; $\mathrm{A} \beta \mathrm{PP}, \beta$-amyloid precursor protein; XAS, X-ray Absorption Spectroscopy; EMBL, European Molecular Biology Laboratory; DESY, Deutsches Elektronen-Synchrotron; EXAFS, Extended X-ray Absorption Fine Structure; MDB, Metallo-protein Database and Browser; MS, multiple scattering; XANES, X-ray absorption near edge; PDB, Protein Data Bank; FT, Fourier Transform.

${ }^{2}$ The total absorption coefficient, $\mu(k)$, is the quantity actually measured, from which the structural signal, $\chi(\boldsymbol{k})$, is defined as the relative oscillations, with respect to the absorption coefficient, $\mu_{0}(k)$, of the isolated absorber $(\mathrm{Cu}$ or $\mathrm{Zn})$. In formulae

$$
\chi(k)=\frac{\mu(k)-\mu_{0}(k)}{\mu_{0}(k)}
$$

where $k$ is the photo-electron wave vector which is related to the incident photon energy $E$ and the ionization energy $E_{0}$ by the obvious relation

$$
\boldsymbol{k}=\sqrt{\frac{2 \boldsymbol{m}\left(E-E_{0}\right)}{\hbar^{2}}},
$$

with $m$ the electron mass and $\hbar$ the Planck constant. 
${ }^{3}$ We recall that this is precisely the situation one encounters when Histidine residues are bound to the absorber. The non negligible MS contributions due to presence of an imidazole ring can be usefully exploited to get a clear-cut determination of the number of Histidine residues directly bound to the metal (Meneghini and Morante 1998; Morante et al. 2004).

${ }^{4}$ According to (Binsted et al. 1992) the number of independent data points, $N_{\text {ind }}$, is defined as

$$
N_{\text {ind }}=\frac{2 \Delta k \Delta r}{\pi}+j
$$

where $\Delta \boldsymbol{k}=\boldsymbol{k}_{\max }-\boldsymbol{k}_{\text {min }}$ is the interval of momenta over which data have been taken, $\Delta \boldsymbol{r}$ is the width of the shell containing all the scatters that are taken into account in the fit and $\boldsymbol{j}$ is a small positive integer not larger than 2 which to be conservative we take to be equal to zero.

${ }^{5} \mathrm{~A}$ critical role in the oxidative stress and neuro-toxicity induced by $\mathrm{A} \beta$-peptides has been attributed to Methionine (Butterfield and Boyd-Kimball 2005).

${ }^{6}$ For completeness and to fix our notations we recall how the parameters introduced in the text enter the formula for the measured absorption coefficient. For simplicity we report formulae valid in the single scattering approximation. In this case the theoretical EXAFS signal has the expression

$$
\chi(\boldsymbol{k})=\boldsymbol{S}_{0}^{2} \sum_{\boldsymbol{l}} \frac{\boldsymbol{N}_{\boldsymbol{l}}}{\boldsymbol{k} \boldsymbol{r}_{l}^{2}}\left|\boldsymbol{f}_{l}(\boldsymbol{k}, \boldsymbol{\pi})\right| \sin \left(2 \boldsymbol{k} \boldsymbol{r}_{l}+\varphi_{l}(\boldsymbol{k})\right) \boldsymbol{e}^{-2 \sigma_{l}^{2} \boldsymbol{k}^{2}} \boldsymbol{e}^{-2 \boldsymbol{r}_{l} / \lambda(\boldsymbol{k})}
$$

where the sum runs over the different coordination shells around the absorber. $N_{l}$ is the number of scatterers of the $l$-th shell, located at a distance $r_{l}$ from the absorber and $\sigma_{l}^{2}$ is the Debye-Waller factor. $\left|f_{l}(k, \pi)\right|$ is the modulus of the back-scattering amplitude and $\varphi_{l}(k)$ the total scattering phase. 
Finally $S_{0}^{2}$ is an empirical quantity that accounts for all the many-body losses in photo-absorption processes and $\lambda(\boldsymbol{k})$ is the photo-electron mean free path. For MS processes a formally similar expression can be derived, in which $r_{l}$ represents the length of the full MS path. Modulus and phase functions are now more complicated expressions which depend on each scattering event along the MS path (Lee and Pendry 1975; Benfatto et al. 1986; Gurman et al. 1986; Koningsberger and Prins 1988; Rehr and Albers 1990).

${ }^{7}$ The $R$-factor of the fit is computed as follows

$$
\boldsymbol{R}=\sum_{i=1}^{P} \frac{1}{\boldsymbol{w}_{i}}\left|\chi^{\exp }\left(\boldsymbol{k}_{\boldsymbol{i}}\right)-\chi^{f i t}\left(\boldsymbol{k}_{\boldsymbol{i}}\right)\right|
$$

where $\chi^{\exp }$ and $\chi^{\text {fit }}$ are the experimental and theoretical data, respectively, and the sum is over the number, $\boldsymbol{P}$, of the $k$ values at which data were collected. The "weighting" parameter $\boldsymbol{w}_{\boldsymbol{i}}$ is defined by the formula

$$
\boldsymbol{w}_{i}=\frac{1}{\boldsymbol{k}_{i}^{n}} \sum_{j=1}^{P} \boldsymbol{k}_{j}^{n}\left|\chi^{\exp }\left(\boldsymbol{k}_{j}\right)\right|
$$

where the integer $n$ is selected in such a way that the amplitude of the EXAFS oscillations in $\boldsymbol{k}^{\boldsymbol{n}} \chi^{\exp }(\boldsymbol{k})$ do not die away at large values of $k$. In this paper we took $\boldsymbol{n}=3$. It is a consolidated experience that for complex biological molecules a fit can be considered adequately good for values of $R$ in the interval between 20\% and 40\% (Binsted 1998).

${ }^{8}$ Error on angles are somewhat large and are of the order of $20 \%$. 


\section{REFERENCES}

Benfatto M, Natoli CR, Bianconi A, Garcia J, Marcelli A, Fanfoni M, Davoli I (1986) Multiplescattering regime and higher-order correlations in x-ray-absorption spectra of liquid solutions. Phys Rev B 34: 5774-5781

Bianconi A, Congiu-Castellano A, Dell'Ariccia M, Giovannelli A, Morante S, Burattini E, Durham PJ (1986) Local Fe site structure in the tense-to-relaxed transition in carp deoxyhemoglobin: a XANES (x-ray absorption near edge structure) study. Proc Natl Acad Sci U.S.A. 83: 7736-7740

Binsted N, Strange RW, Hasnain SS (1992) Constrained and restrained refinement in EXAFS data analysis with curved wave theory. Biochemistry 31: 12117-12125

Binsted N (1998) EXCURV98: CCLRC Daresbury Laboratory computer program.

Bush AI (2000) Metals and neuroscience. Curr Opin Chem Biol 4: 184-191

Butterfield DA, Boyd-Kimball D (2005) The critical role of methionine 35 in Alzheimer's amyloid beta-peptide (1-42)-induced oxidative stress and neurotoxicity. Biochim Biophys Acta 1703: 149156

Castagnetto JM, Hennessy SW, Roberts VA, Getzoff ED, Tainer JA, Pique ME (2002) MDB: the Metalloprotein Database and Browser at The Scripps Research Institute. Nucleic Acids Res 30: 379-382

Cherny RA, Atwood CS, Xilinas ME, Gray DN, Jones WD, McLean CA, Barnham KJ, Lynch T, Volitakis I, Fraser FW, Kim YS, Huang X, Goldstein LE, Moir RD, Lim JT, Beyreuther K, Zheng H, Tanzi RE, Masters CL, Bush AI (2001) Treatment with a copper-zinc chelator markedly and rapidly inhibits beta-amyloid accumulation in Alzheimer's disease transgenic mice. Neuron 30: 665-676

Cheung KC, Strange RW, Hasnaina SS (2000) 3D EXAFS refinement of the $\mathrm{Cu}$ site of azurin sheds light on the nature of structural change at the metal centre in an oxidation \pm reduction process: an integrated approach combining EXAFS and crystallography. Acta Cryst D56: 697-704

Comai M, Dalla Serra M, Potrich C, Menestrina G (2003) $\mathrm{Cu}^{2+}$ and $\mathrm{Zn}^{2+}$ effects on beta-amyloid aggregation and structural conformation. Biophys J 84: 337a

Curtain CC, Ali F, Volitakis I, Cherny RA, Norton RS, Beyreuther K, Barrow CJ, Masters CL, Bush AI, Barnham KJ (2001) Alzheimer's disease amyloid-beta binds copper and zinc to generate an allosterically ordered membrane-penetrating structure containing superoxide dismutase-like subunits. J Biol Chem 276: 20466-20473 
D’Angelo P, Benfatto M, Della Longa S, Pavel NV (2002) Evidence of distorted fivefold coordination of the $\mathrm{Cu} 2+$ aqua ion from an x-ray absorption spectroscopy quantitative analysis. Phys Rev B 66: 1-7

Finefrock AE, Bush AI, Doraiswamy PM (2003) Current status of metals as therapeutic targets in Alzheimer's disease. J Am Geriatr Soc 51: 1143-1148

Gurman SJ, Binsted N, Ross I (1986) A rapid, exact, curved-wave theory for EXAFS calculations. II. The multiple-scattering contributions. J Phys C 19: 1845-1861

Hasnain SS, Murphy LM, Strange RW, Grossmann JG, Clarke AR, Jackson GS, Collinge J (2001) XAFS study of the high-affinity copper-binding site of human $\operatorname{PrP}(91-231)$ and its low-resolution structure in solution. J Mol Biol 311: 467-473

Huang X, Atwood CS, Moir RD, Hartshorn MA, Vonsattel JP, Tanzi RE, Bush AI (1977) Zincinduced Alzheimer's A $\beta 1-40$ Aggregation Is Mediated by Conformational Factors. J Biol Chem 272: $26464-26470$

Karr JW, Kaupp LJ, Szalai VA (2004) Amyloid-beta binds Cu2+ in a mononuclear metal ion binding site. J Am Chem Soc 126: 13534-13538

For a review see: Koningsberger DC, Prins R, editors "X-ray Absorption. Principles, applications, techniques of EXAFS, SEXAFS and XANES", John Wiley \& Sons (1988), and references quoted therein.

Kowalik-Jankowska T, Ruta M, Wiśniewska K, Lankiewicz L (2003) Coordination abilities of the 1-16 and 1-28 fragments of $\beta$-amyloid peptide towards copper(II) ions: a combined potentiometric and spectroscopic study. J Inorg Biochem 95: 270-282

Lee PA, Pendry JB (1975) Theory of the extended x-ray absorption fine structure. Phys Rev B 11: 2795-2811

Lee PA, Citrin PH, Eisenberg P, Kinkaid BM (1981) Extended x-ray absorption fine structure-its strengths and limitations as a structural tool. Rev Mod Phys 53: 769-806

Meneghini C, Morante S (1998) The active site structure of tetanus neurotoxin resolved by multiple scattering analysis in X-Ray absorption spectroscopy. Biophys J 75: 1953-1963

Miura T, Suzuki K, Kohata N, Takeuchi H (2000) Metal Binding Modes of Alzheimer's Amyloid $\beta$-Peptide in Insoluble Aggregates and Soluble Complexes. Biochemistry 39: 7024-7031

Morante S, Gonzalez-Iglesias R, Potrich C, Meneghini C, Meyer-Klaucke W, Menestrina G, Gasset $\mathrm{M}$ (2004) Inter- and intra-octarepeat $\mathrm{Cu}$ (II) site geometries in the prion protein: implications in $\mathrm{Cu}$ (II) binding cooperativity and $\mathrm{Cu}(\mathrm{II})$-mediated assemblies. J Biol Chem 279: 11753-11759

Natoli CR, Benfatto M (1986) A unifying scheme of interpretation of X-ray absorption spectra based on multiple scattering theory. J Phys (France) Colloq C8 47: $11-23$ 
Nolting HF, Hermes C (1992) EXPROG: EMBL EXAFS data analysis and evaluation program package for PC/AT.

Opazo C, Barria MI, Ruiz FH, Inestrosa NC (2003) Copper reduction by copper binding proteins and its relation to neurodegenerative diseases. Biometals 16: 91-98

Pepys MB (2001) Pathogenesis, diagnosis and treatment of systemic amyloidosis. Philos Trans R Soc Lond B Biol Sci 356: 203-210

Redecke L, Meyer-Klaucke W, Koker M, Clos J, Georgieva D, Genov N, Echner H, Kalbacher H, Perbandt M, Bredehorst R, Voelter W, Betzel C (2005) Comparative analysis of the human and chicken prion protein copper binding regions at pH 6.5. J Biol Chem 280: 13987-13992

Rehr JJ, Albers RC (1990) Scattering-matrix formulation of curved-wave multiple-scattering theory: Application to x-ray-absorption fine structure. Phys Rev B 41: 8139-8149

Selkoe DJ (2001) Alzheimer's disease: genes, proteins, and therapy. Physiol Rev 81: 741-766

Syme CD, Nadal RC, Rigby SEJ, Viles JH (2004) Copper Binding to the Amyloid- $\beta$ (A $\beta$ ) Peptide Associated with Alzheimer's Disease. J Biol Chem 279: 18169-18177

Suzuki K, Miura T, Takeuchi H (2001) Inhibitory Effect of Copper(II) on Zinc(II)-Induced Aggregation of Amyloid $\beta$-Peptide. Biochem Biophys Res Commun 285: 991-996

Teo BK, Lee PA (1979) Ab initio calculations of amplitude and phase functions for extended x-ray absorption fine structure spectroscopy J Am Chem Soc 101: 2815-2832

Tjernberg LO, Callaway DJ, Tjernberg A, Hahne S, Lilliehook C, Terenius L, Thyberg J, Nordstedt C (1999) A molecular model of Alzheimer amyloid beta-peptide fibril formation. J Biol Chem 274: $12619-12625$

Tyson TA, Hodgson KO, Natoli CR, Benfatto M (1992) General multiple-scattering scheme for the computation and interpretation of $\mathrm{x}$-ray-absorption fine structure in atomic clusters with applications to SF6, GeCl4, and Br2 molecules. Phys Rev B 46: 5997-6019

\section{TABLE LEGEND}

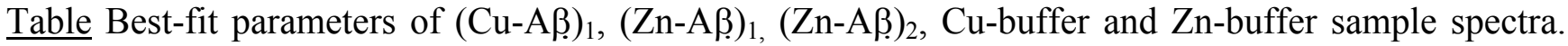
First and second column: type and number of coordinated chemical group (atom or residue); third column: distance from the absorber of the coordinated atom or of the leading atom of the coordinated residue; fourth column: value of the corresponding DW factor, $\sigma_{\boldsymbol{D} W}^{2}$. Statistical errors are given next to each parameter. In the last row we report the Fermi energy shift $\Delta E_{f}$ and the $R$-factor of the fit, as well as the number of independent data points and fitted parameters. 


\section{FIGURE LEGEND}

Fig.1 Absorption coefficient in the XANES region of the spectrum of: $-(\mathrm{Cu}-\mathrm{A} \beta)_{1}$, $\mathrm{A} \beta$-peptide complexed with $\mathrm{Cu}^{2+}$ in solution (dotted line); $\bullet(\mathrm{Zn}-\mathrm{A} \beta)_{1}, \mathrm{~A} \beta$-peptide complexed with $\mathrm{Zn}^{2+}$ in solution (broken line); $\bullet(\mathrm{Zn}-\mathrm{A} \beta)_{2}, \mathrm{~A} \beta$-peptide complexed with $\mathrm{Zn}^{2+}$ in the re-dissolved pellet (continuous line). To ease comparison, spectra are shifted in energy (by about $680 \mathrm{eV}$ ) in order for the $\mathrm{K}$-edges of $\mathrm{Cu}^{2+}$ and $\mathrm{Zn}^{2+}$ to be located at almost the same energy. The energy scale is given with reference to the edge energy of the absorbers. The latter is empirically taken as the energy where the first derivative of the absorption spectrum attains its maximum.

Fig.2 Absorption coefficient in the XANES region of the spectrum of the three $\mathrm{Zn}^{2+}$ samples: $(\mathrm{Zn}-\mathrm{A} \beta)_{1}$ (dotted line), $(\mathrm{Zn}-\mathrm{A} \beta)_{2}$ (broken line) and $\mathrm{Zn}$-buffer (continuous line). The energy scale is set as in Fig.1.

Fig.3 EXAFS structural signal of the $(\mathrm{Cu}-\mathrm{A} \beta)_{1}$ complex. The simulated signal (black line) is drawn superimposed to the experimental data (gray line). The experimental data as well as the simulated signal have been multiplied by $\boldsymbol{k}^{3}$.

Fig.4 EXAFS structural signal of (a) the $(\mathrm{Zn}-\mathrm{A} \beta)_{1}$ and (b) the $(\mathrm{Zn}-\mathrm{A} \beta)_{2}$ complex. The simulated signal (black line) is drawn superimposed to the experimental data (gray line). The experimental data as well as the simulated signal have been multiplied by $\boldsymbol{k}^{3}$.

Fig.5 Schematic pictures of the molecular arrangement around the absorber. Panel (a) is for the $(\mathrm{Cu}-$ $\mathrm{A} \beta)_{1}$ sample; $(\mathrm{b})$ for $(\mathrm{Zn}-\mathrm{A} \beta)_{1}$ and $(\mathrm{c})$ for $(\mathrm{Zn}-\mathrm{A} \beta)_{2}$. The PDB file is directly generated by EXCURV98. Atom colors are as follows: copper $(\mathrm{Cu})$ green; zinc $(\mathrm{Zn})$ black; oxygen $(\mathrm{O})$ red; nitrogen $(\mathrm{N})$ blue; carbon $(\mathrm{C})$ gray; chlorine, $(\mathrm{Cl})$ light blue. The following abbreviations are used for the residues bound to the absorber: His for Histidine; Tyr for Tyrosine.

Fig.6 Fourier transforms (FT) of the EXAFS structural signal of the three samples, $(\mathrm{Cu}-\mathrm{A} \beta)_{1},(\mathrm{Zn}-\mathrm{A} \beta)_{1}$, and $(\mathrm{Zn}-\mathrm{A} \beta)_{2}$ (panels $\mathrm{a}, \mathrm{b}$ and $\mathrm{c}$, respectively). FT's of experimental data (gray line) are drawn superimposed to the FT's of the theoretical spectra (black line).

Fig.7 Schematic illustration of the structures of $(\mathrm{Cu}-\mathrm{A} \beta)_{1}$ (panel a), $(\mathrm{Zn}-\mathrm{A} \beta)_{1}$ (panel b) and $(\mathrm{Zn}-\mathrm{A} \beta)_{2}$ (panel c), as they emerge form the fit. Only Metal-Histidine bonds are explicitly drawn. The $\mathrm{A} \beta$-peptide backbone is drawn as a shoe string. 
Table Best fit parameters

\begin{tabular}{|c|c|c|c|}
\hline $\begin{array}{c}\text { Coordinated } \\
\text { residue }\end{array}$ & $N$ & $r \pm \delta r(\AA)$ & $\sigma_{D W}^{2} \pm \delta \sigma_{D W}^{2}\left(\AA^{2}\right)$ \\
\hline \multicolumn{4}{|c|}{$(\mathrm{Cu}-\mathrm{A} \beta)_{1}=\mathrm{Cu}-\mathrm{A} \beta_{1-40^{-}-\text {solution }}$} \\
\hline $\operatorname{His}^{(a)}$ & 2 & $1.94 \pm 0.01$ & $0.008 \pm 0.003$ \\
\hline $\mathrm{His}^{(\mathrm{b})}$ & 1 & $1.85 \pm 0.01$ & $0.009 \pm 0.004$ \\
\hline Tyr & 1 & $2.00 \pm 0.01$ & $0.003 \pm 0.001$ \\
\hline \multirow[t]{2}{*}{$\mathrm{O}^{(\mathrm{c})}$} & 1 & $1.91 \pm 0.02$ & $0.004 \pm 0.002$ \\
\hline & $8 \pm 0.4 \mathrm{eV}$ & $R=32 \%$ & $N_{\text {par }}=9 \quad N_{\text {ind }}=17$ \\
\hline \multicolumn{4}{|c|}{$(\mathrm{Zn}-\mathrm{A} \beta)_{1}=\mathrm{Zn}-\mathrm{A} \beta_{1-40}$-solution } \\
\hline His & 1 & $2.20 \pm 0.02$ & $0.004 \pm 0.001$ \\
\hline Tyr & 1 & $1.99 \pm 0.02$ & $0.005 \pm 0.001$ \\
\hline $\mathrm{O}^{(\mathrm{c})}$ & 2 & $2.01 \pm 0.02$ & $0.011 \pm 0.001$ \\
\hline \multirow[t]{2}{*}{$\mathrm{Cl}$} & 2 & $2.26 \pm 0.01$ & $0.005 \pm 0.001$ \\
\hline & $.1 \pm 0.4 \mathrm{eV}$ & $R=29 \%$ & $N_{\text {par }}=9 \quad N_{\text {ind }}=19$ \\
\hline
\end{tabular}

$(\mathrm{Zn}-\mathrm{A} \beta)_{2}=\mathrm{Zn}-\mathrm{A} \beta_{1-40}-$ pellet

\begin{tabular}{|c|c|c|c|}
\hline His & 2 & $2.00 \pm 0.01$ & $0.004 \pm 0.002$ \\
\hline Tyr & 1 & $2.00 \pm 0.03$ & $0.012 \pm 0.008$ \\
\hline $\mathrm{O}^{(\mathrm{c})}$ & 3 & $1.98 \pm 0.03$ & $0.010 \pm 0.008$ \\
\hline & $\Delta E_{f}=-8.2 \pm 0.4 \mathrm{eV}$ & $R=27 \%$ & $N_{\text {ind }}=16$ \\
\hline
\end{tabular}

\section{Cu-buffer}

\begin{tabular}{|c|c|c|c|}
\hline Op & 4 & $1.94 \pm 0.01$ & $0.005 \pm 0.001$ \\
\hline $\mathrm{Oa}$ & 2 & $2.79 \pm 0.02$ & $0.011 \pm 0.001$ \\
\hline \multicolumn{3}{|c|}{$\Delta E_{f}=-9.8 \pm 0.3 \mathrm{eV}$} & \\
\hline
\end{tabular}

\section{Zn-buffer}

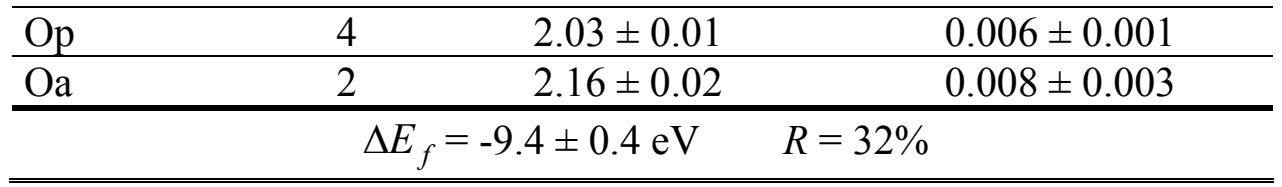

(a) The distance, $r$, refers to the position of the "leading" residue atom (see text). This is $\mathrm{N}$ for the Histidine residue and $\mathrm{O}$ for the Tyrosine.

(b) Histidine residues whose normal to imidazole planes have (significantly) different orientation are reported separately (see text).

(c) This $\mathrm{O}$ belongs to a water molecule or to amino acidic residues other than the coordinated Histidine and Tyrosine residues (see text). 


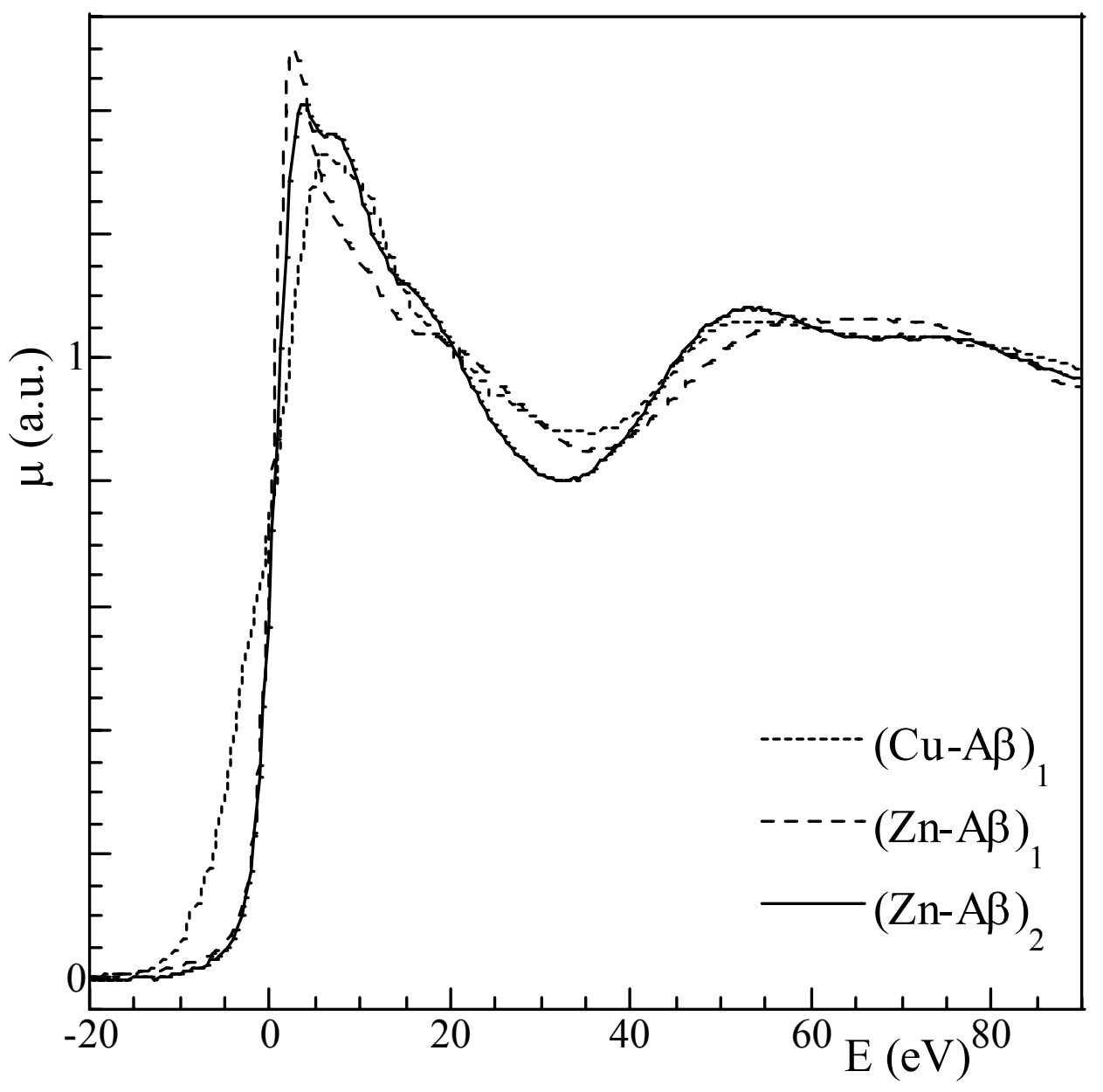

Figure 1 


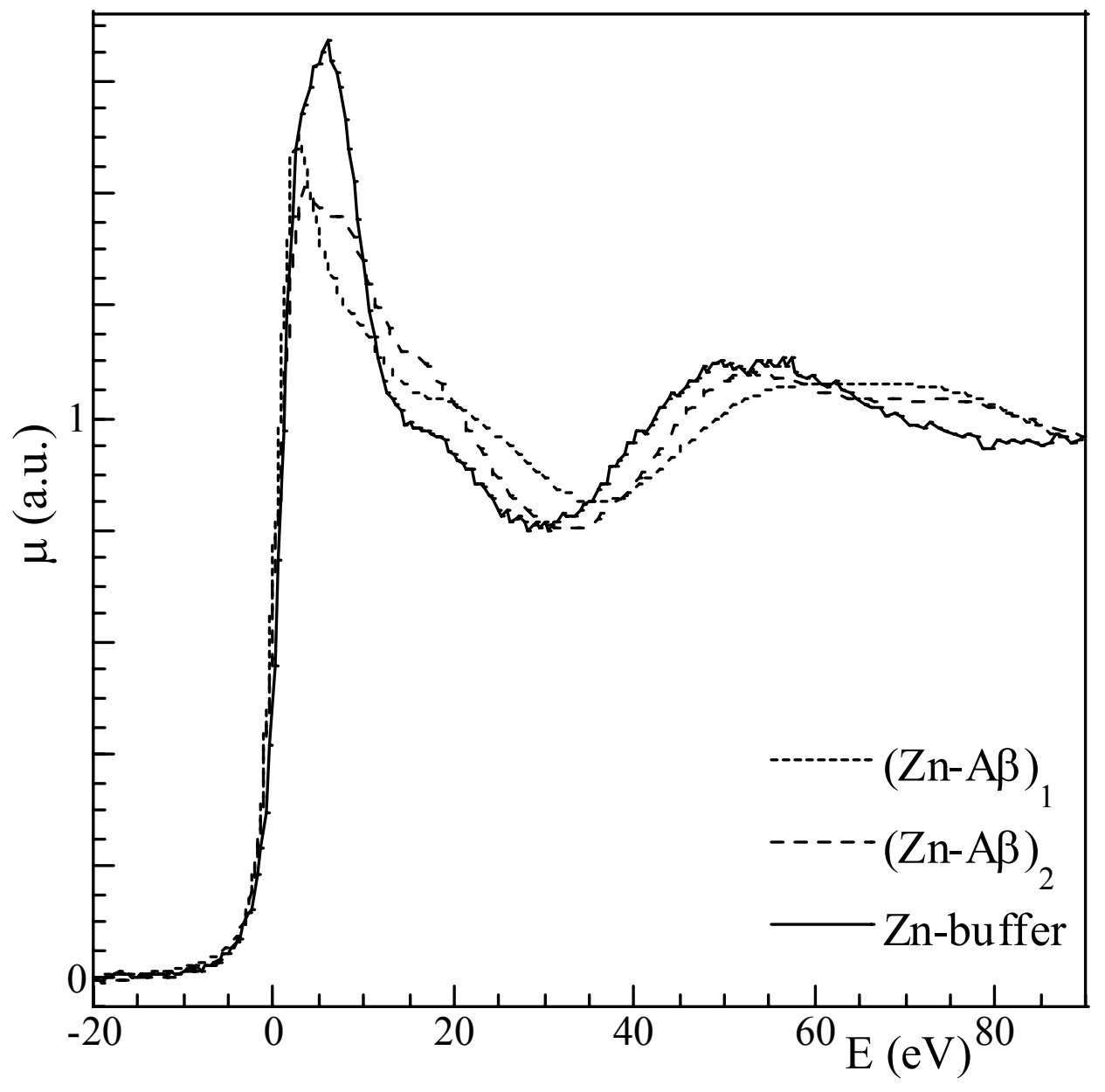

Figure 2 


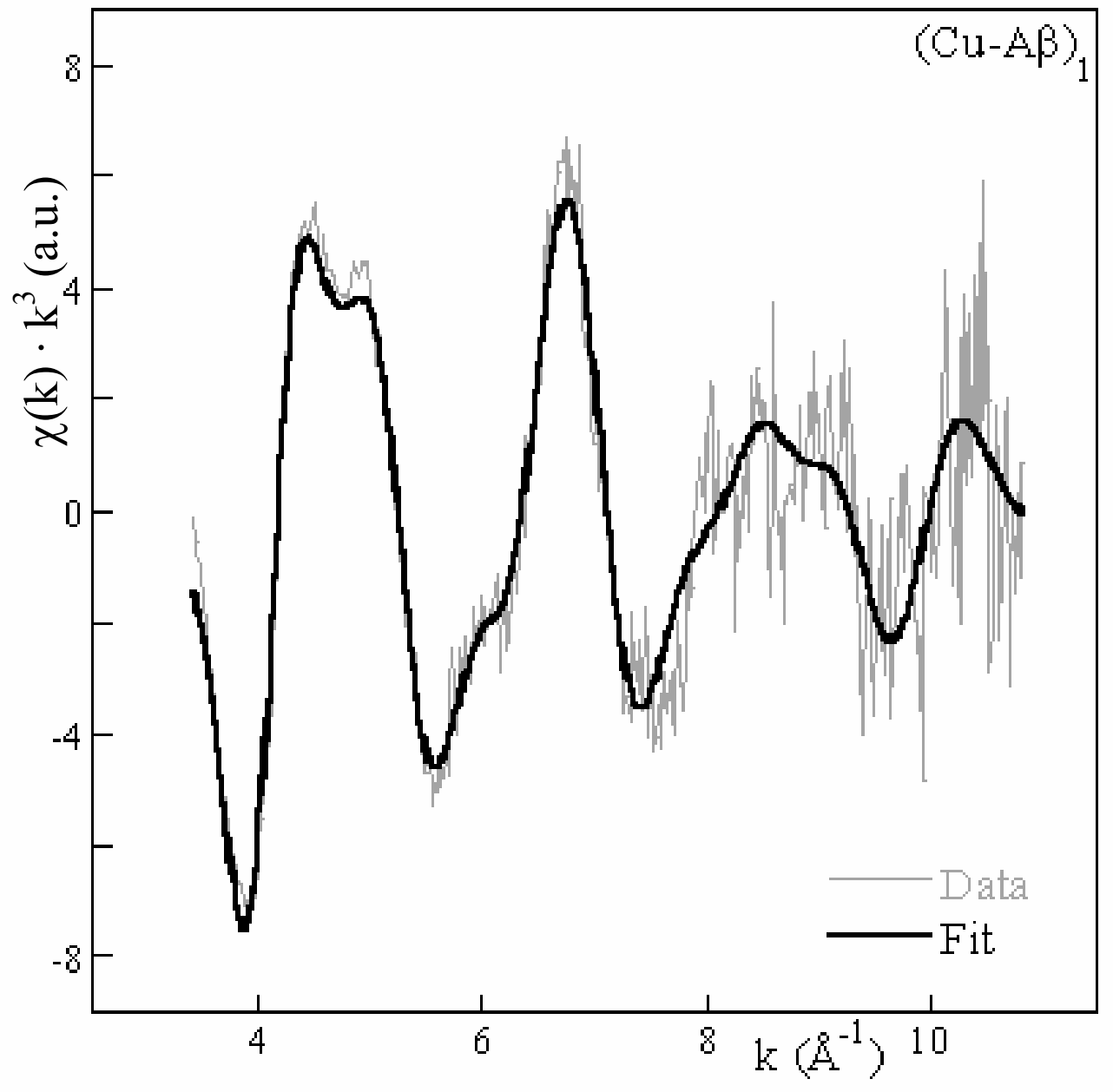

Figure 3 


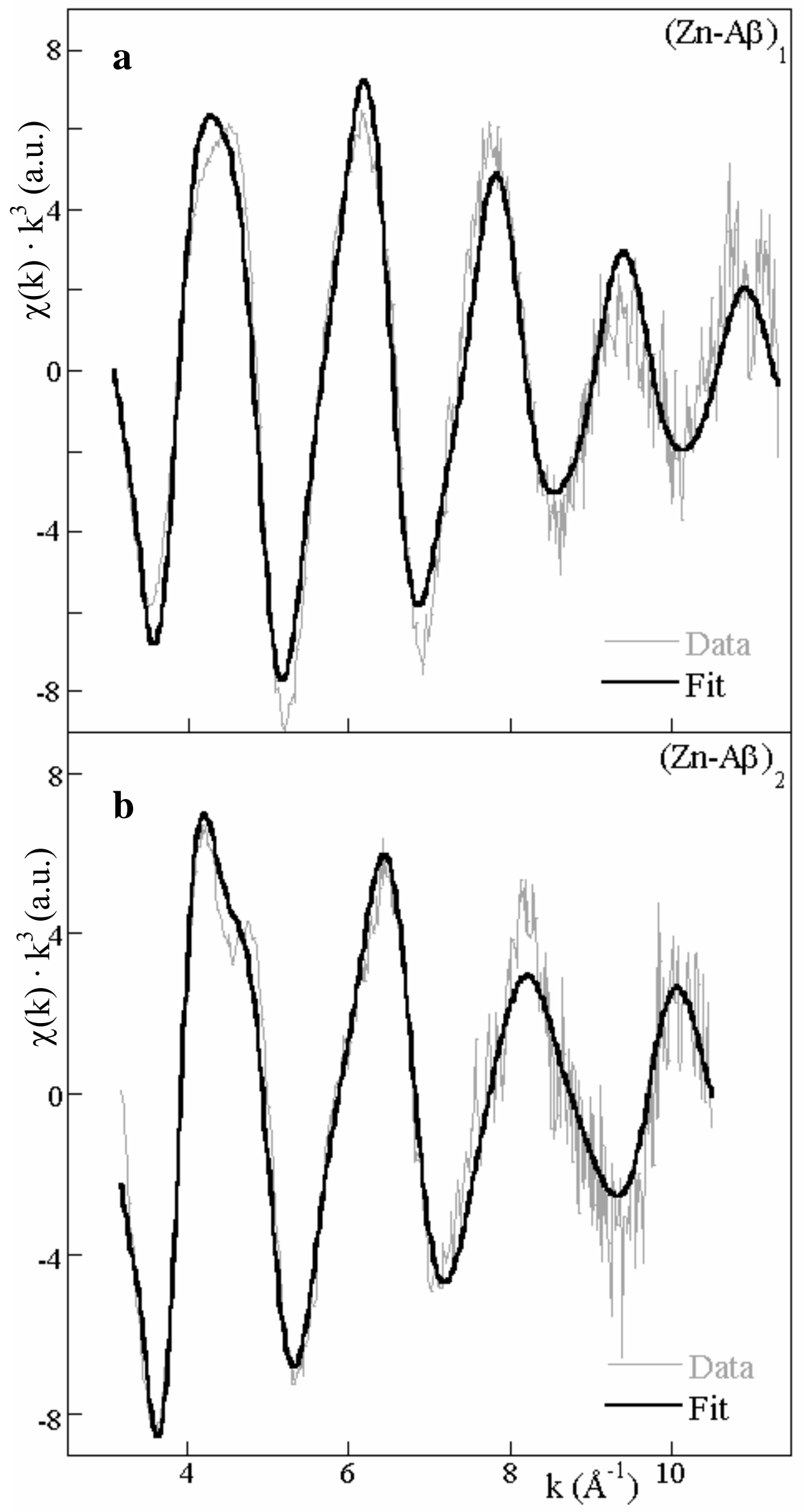

Figure 4 


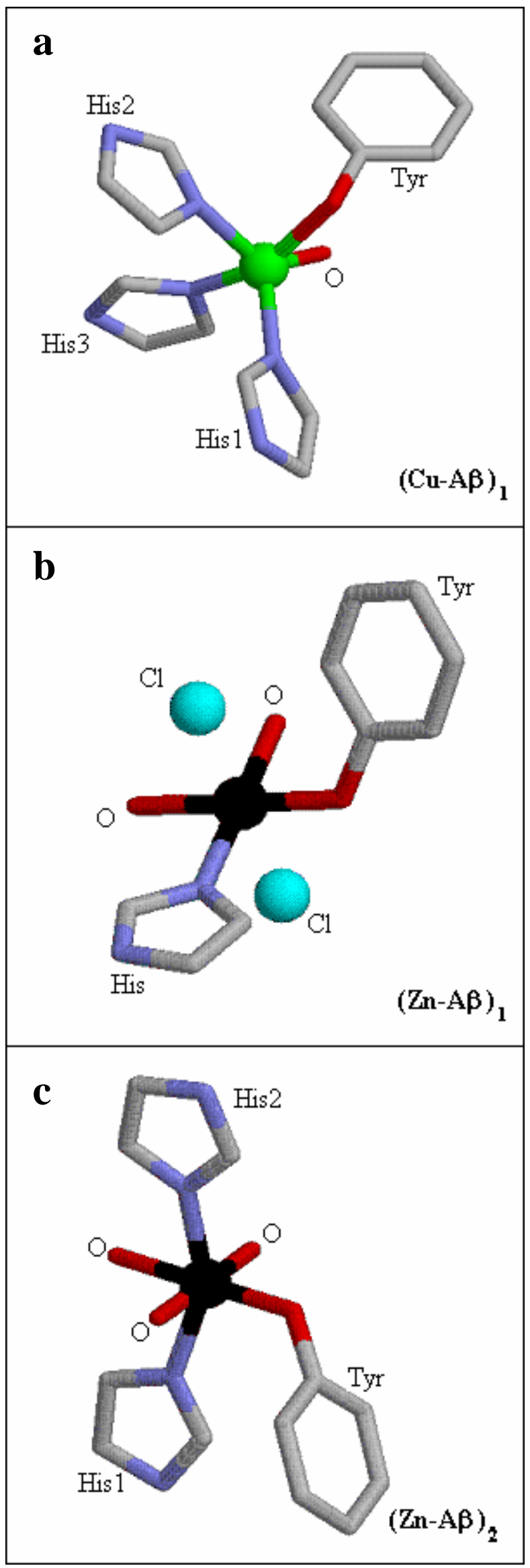

Figure 5 


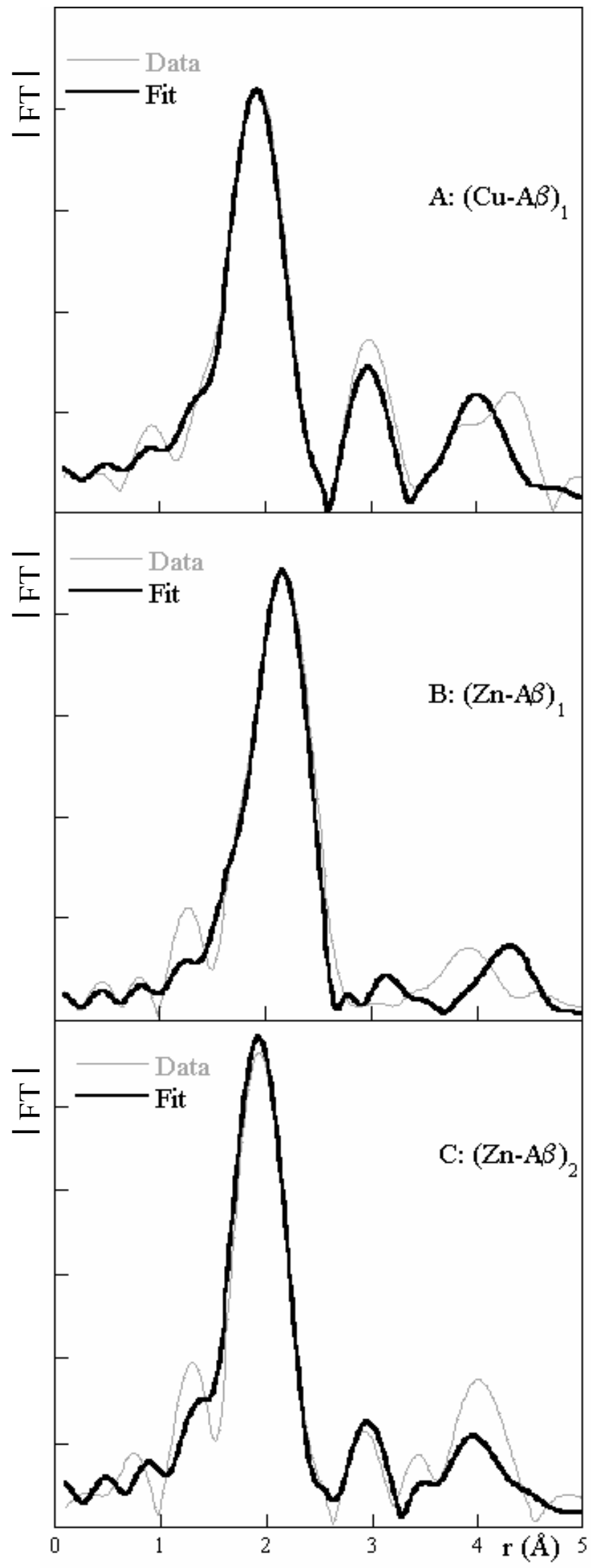

Figure 6 


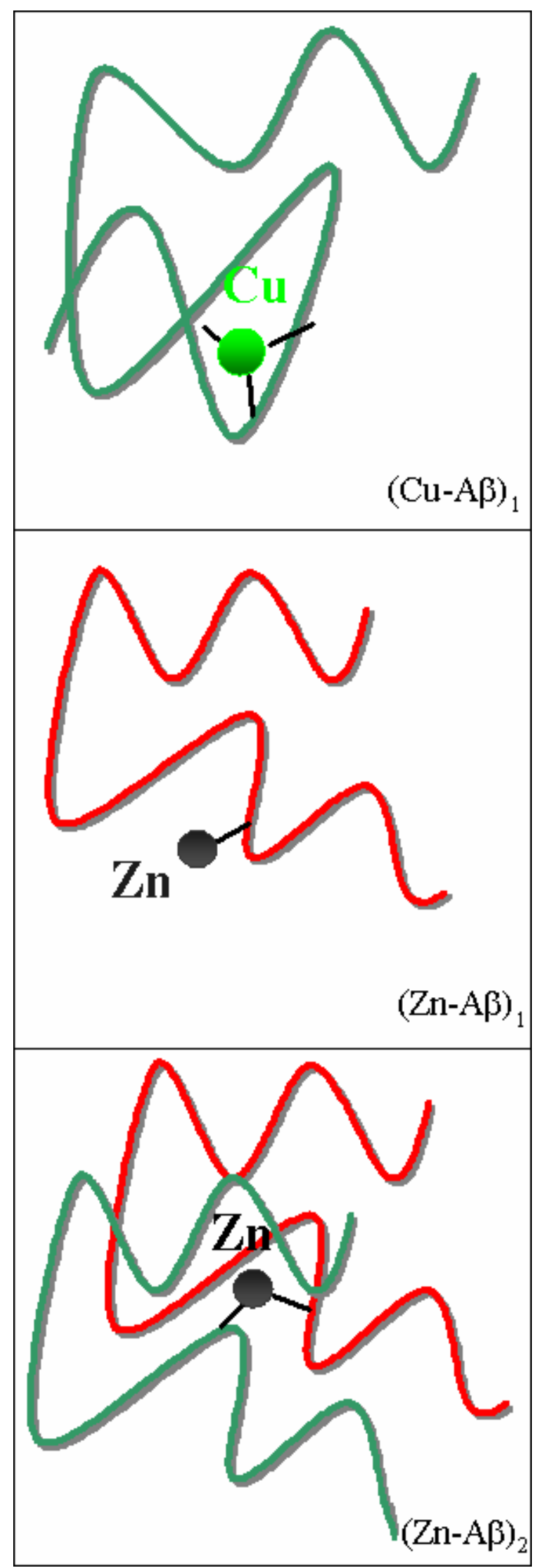

Figure 7 